Running head: ENCOUNTER EXPECTANCY BIAS IN FEAR OF SPIDERS

Evidence For an Encounter Expectancy Bias in Fear of Spiders

\author{
Tatjana Aue and Marie-Eve Hoeppli \\ University of Geneva, Geneva, Switzerland
}

Address correspondence to:

Tatjana Aue

Swiss Center for Affective Sciences

7, Rue des Battoirs

1205 Geneva

Switzerland

tatjana.aue@unige.ch

Phone: +41223795979 


\begin{abstract}
Whereas research has demonstrated that phobic or fearful individuals overestimate the likelihood to incur aversive consequences from an encounter with feared stimuli, it has not yet been systematically investigated whether these individuals also overestimate the likelihood (i.e., the frequency) of such encounters. In the current experiment, spider fearful and control participants were presented with background information that allowed them to estimate the overall likelihood that different kinds of animals (spiders, snakes, or birds) would be encountered. Spider fearful participants systematically overestimated the likelihood of encountering a spider with respect to the likelihood of encountering a snake or a bird. No such expectancy bias was observed in control participants. The results thus strengthen our idea that there exist indeed two different types of expectancy bias in high fear and phobia that can be related to different components of the fear response. A conscientious distinction and examination of these two types of expectancy bias are of potential interest for therapeutic applications.
\end{abstract}

Keywords: fear of spiders, expectancy bias, risk of encounter, ratings 


\section{Evidence For an Encounter Expectancy Bias in Fear of Spiders}

Biased cognitive processing in anxiety and fear includes selective abstraction and interpretation (e.g., Huijding \& de Jong, 2007). It can lead to hypersensitivity for harmful features in a given situation, while largely ignoring its positive aspects, and therefore to the underestimation of personal coping potential and the availability of safety cues (Beck \& Clark, 1997). Related to this issue, spider fearful individuals have been reported to not only overestimate the likelihood of negative consequences of spider presentations (i.e., losing candy after having seen a spider) but also to underestimate the likelihood of positive consequences of spider presentations (i.e., winning candy after having seen a spider; Muris, Huijding, Mayer, den Breejen, \& Makkelie, 2007). Thus, a critical feature of pathological anxiety or fear consists of the exaggerated experience of threat that is not justified by the internal and external environments. Non-clinical anxiety and fear, on the contrary, have been suggested to better correspond to actual, objective, danger (Beck \& Clark, 1997).

Particularly interesting are cognitive biases related to evolutionary fear-relevant stimuli, such as snakes and spiders (see Davey, 1995; Davey \& Dixon, 1996, for an overview). When asked after a study, participants overestimated the frequency of having received an electric shock after the presentation of such fear-relevant as compared with fear-irrelevant pictures ( $a$ posteriori expectancy bias or covariation bias [as opposed to the a priori expectancy bias, which refers to biased expectations before study onset]; Tomarken, Mineka, \& Cook, 1989). Interestingly this overestimation was limited to phylogenetic threat stimuli and was not obtained with ontogenetic threat stimuli such as weapons (de Jong et al., 1992). Mühlberger, Wiedemann, Herrmann, and Pauli (2006) showed that the a priori expectancy bias did not differ between phylogenetic phobia (spider phobia) and ontogenetic phobia (flight phobia). Both spider phobics and flight phobics exhibited an overestimation of the likelihood that their particularly feared type of threat would be 
followed by a startling white noise (cf. de Jong \& Merckelbach, 1991; de Jong et al., 1992). However, the correction of the a priori expectancy bias throughout the experiment was slower in phylogenetic as compared with ontogenetic phobia. Together, these observations point to the great importance of the study of a-priori expectancies that are characteristic for evolutionrelevant types of fear (e.g., spider phobia).

Despite the generally accepted claim that spider phobics display exaggerated negative apriori expectations towards threat, the concrete contents of these expectations have not been clearly classified. We postulate that it is worth distinguishing exaggerated expectations concerning the likelihood of an encounter with a feared stimulus (encounter expectancy bias) from exaggerated expectations concerning the likelihood of incurring aversive consequences of such an encounter (consequences expectancy bias). For example, a study by Dijk and de Jong (2009) demonstrated that blushing phobics did not overestimate the negative consequences of blushing. However, at the same time, these phobics expected to blush more often than did individuals who were characterized by lower levels of fear of blushing.

From a biological preparedness perspective, it would seem to be adaptive to overestimate the likelihood of encountering a stimulus if we overestimate the harmful consequences associated with this stimulus. This is because the encounter expectancy bias would ensure the initiation of compensatory actions (e.g., avoidance) even in situations where there is little risk of an encounter. Such response preparation, in the long run, should decrease the actual likelihood of an encounter and, as a consequence, also the likelihood to suffer from harm. Thus, the idea here is not that the expectancy bias for encounters is completely independent from the expectancy bias for negative consequences stemming from an encounter. By contrast, the overestimation of the subjective likelihood to encounter a dangerous animal may very well vary as a function of its 
anticipated negative consequences. But this does by no means imply that the investigation of anxiety and phobia can be restricted to the study of negative consequences only.

Although the proposed distinction may seem trivial at first glance, the two types of expectancy bias should have different implications with respect to experienced fear. Whereas, in everyday life, a phobic individual's overestimation of the likelihood or severity of aversive consequences arising from an encounter with, for instance, a spider is most likely to influence fear intensity, the overestimation of the likelihood of an encounter should be related to the likelihood of occurrence (i.e., the frequency of occurrence) of fear episodes.

The above-cited research on expectancy bias focused more closely on the likelihood to incur negative consequences in phobia- or fear-relevant situations (consequences expectancy bias). There is, however, at least some indirect indication that there might indeed be an expectancy bias for encounters as well. With a signal detection paradigm, Becker and Rinck (2004) investigated whether spider fearful individuals were more sensitive to spiders, beetles, and butterflies than non-fearful individuals. Spider fearful participants were characterized by more liberal response criterions for spiders (but also for beetles) than were non-fearful participants, but they did not display greater sensitivity. These results point out, that fearful individuals have a greater tendency to interpret ambiguous or uncertain situations as fear-related. Such an interpretation bias may well be the result of heightened expectations of encountering the feared stimulus. Comparably, after actual encounters with feared stimulus material, a memory bias has been observed (Watts \& Dalgleish, 1991). Spider fearful individuals tended to falsely recall more spider-related words than neutral words (words that had not actually been presented before). On the other hand, the Mühlberger et al. (2006) study with spider and flight phobics did not reveal any difference in picture frequency estimates for phobic versus nonphobic content. 
A first study addressing encounter expectancies more explicitly (although this was not the central aim of the study) was conducted by de Jong and Muris (2002), who demonstrated that spider phobia was positively related to the expectation of spiders entering a room or of these being present in a home environment. Since, in this study, encounter ratings were restricted to spiders, it remains to be investigated whether heightened expectations of encounter in spider fearful or phobic individuals exist for spiders only, or whether they generalize to other animals. Moreover, to date, these individuals' encounter expectations for places outside the home environment have not been investigated. The study of expectations outside the home environment is of importance because it allows examining whether encounter expectancies are more general in nature or restricted to the home environment.

Finally, it remains to be examined whether heightened expectancies of encounter do exist when spider fearful and phobic individuals are pre-informed about a potential risk of encounter. It is possible that they ignore such information and focus on their "gut feeling" instead, consequently making the expectancy bias particularly evident when the risk of encounter suggested by background information is very low.

In the current study we investigated the a priori encounter expectancy bias. Spider fearful ${ }^{1}$ and non spider fearful participants (control participants) imagined visiting different locations in a forest and were given background information about the number of times two forest officials had encountered different animals at these locations. Our participants rated the subjective risk to encounter the animals in question if they themselves visited the displayed location. It was hypothesized that spider fearful individuals would overestimate the likelihood to encounter spiders but not animals they did not particularly fear (snakes and birds; this is because spider fearful participants were selected on the basis that they displayed high fear of spiders but very low fear of snakes). The control participants were expected to not display such a distinction 
between the different animals (because they were selected on the basis that they displayed particularly low fear of both spiders and snakes).

Moreover, we hypothesized that spider fearful individuals would demonstrate an especially strong overestimation of the risk of encountering spiders when objective background information suggested such encounters to be unlikely. This hypothesis is consistent with the observation of a lower response criterion for spider denominations in the Becker and Rinck (2004) study, which suggests that spider fearful individuals are characterized by intolerance for even slight degrees of uncertainty in potentially fear-relevant situations and therefore opt for the risk interpretation, just to be on the safe side. Such an effect should be particularly visible in low likelihood situations.

\section{Method}

\section{Participants}

36 participants (all female; 18 spider fearful), aged between 19 and 44 years $(M=25.8$, $S D=5.79$ ) were recruited via ads placed in University buildings as well as on University and local websites, looking for participants who were (a) extremely fearful of (including strong physiological responding to and avoidance of) spiders and not fearful of snakes or (b) not fearful of both spiders and snakes. The study was embedded in a larger project investigating decision making, psychophysiological and central nervous responses while imagining encounters with feared and nonfeared animals. The ads explicitly specified these project aims. Persons interested in the study were subjected to a telephone interview and checked for DSM-IV and ICD-10 criteria for presence/absence of spider phobia (adapted from Mühlberger et al., 2006) and low snake fear. Twenty-seven percent of all individuals contacting the experimenters did meet the selection criteria and participated in the study. One participant in the control group was excluded 
from all analyses because she had realized in the middle of the experiment that she did not perform the task correctly.

Apart from meeting/not meeting criteria for spider phobia, fear of spiders and snakes was also assessed by the asking the participants to rate their respective fears on a scale from 0 (no fear at all) to 100 (maximal or extreme fear). Spider fearful individuals rated their fear of spiders much higher than control participants, $t(33)=15.22, p<.001(M \mathrm{~s}=83.6$ and 16.4 , respectively $)$. The two groups did not differ with respect to their ratings for fear of snakes, $t(33)=-0.40, n s(M s$ $=11.2$ and 12.4). Fear of spiders and snakes was further assessed after the experiment by the use of the French translations of the Fear of Spiders Questionnaire (Szymanski \& O’Donohue, $1995), t(33)=9.19, p<.001(M \mathrm{~s}=86.2$ and 23.5) and the Snake Questionnaire (Klorman et al., $1974), t(33)=0.74, n s(M s=3.9$ and 3.2, respectively $)$. Participants in the two groups did not differ with respect to age $t(33)=-0.11, n s(M \mathrm{~s}=25.7$ and 25.9, respectively $)$.

\section{Stimuli}

Stimuli consisted of 30 pictures displaying spiders and 30 pictures displaying snakes, all taken from a recently created picture base (Dan Glauser \& Scherer, in press). Spiders and snakes covered virtually the whole picture and were located on trees, in front of trees, or on the grass. Spider and snake pictures were matched for valence, $t(58)=0.08, n s(M \mathrm{~s}=3.1$ and 3.1 , for spiders and snakes, respectively; scale range: 1 [very unpleasant] - 9 [very pleasant]) and arousal ratings, $t(58)=0.03, n s(M s=6.1$ and 6.1 , for spiders and snakes, respectively; scale range: 1 [not arousing at all] -9 [very arousing]), which had been assessed in an earlier study on an unselected group of University students (Dan Glauser \& Scherer, in press). Thirty additional pictures displaying birds were collected from the internet. Ninety pictures of different forest locations (all of them showing several trees and the ground) preceded the presentation of the animal pictures. Forest locations preceding a specific animal picture varied across participants. 
An additional picture set of 10 neutral animals (e.g., goats and frogs) and 10 forest locations was included for use in ten practice trials.

\section{Procedure}

Upon participants' arrival at the laboratory the nature of the experiment was explained and written informed consent was obtained in accordance with the Helsinki Declaration of Human Rights (1991). Participants were given the occasion to go through 10 practice trials with neutral animals only (e.g., goats and frogs) to get familiar with the task. They imagined that they were visiting different locations in a forest and were told that two forest officials had encountered specific animals (spiders, snakes, or birds) at these same locations for a certain number of times. Participants got information on the frequency of encounters of these two officials with the respective animals. Specifically, in each trial, participants saw a picture of a forest location ( $1 \mathrm{~s})$, followed by a picture of an animal (spider, snake, or bird; $4 \mathrm{~s}$ ). At the time they saw the animals, participants were simultaneously given background information about (1) the number of times the first forest official had encountered a specific animal as related to the number of times he had visited the location (e.g., 2/9) and (2) the number of times the second forest official has encountered this animal as related to the number of times he had visited the location (e.g., 0/9). The two relative frequencies were displayed below the animal pictures. The objective probabilities (= average of the two likelihoods given as background information) were equal across the three animals. The participants' task was to determine the likelihood that they themselves would encounter the animal described when being at the same location in the forest on a 17-point scale (ranging from $0 \%$ [no risk of encounter at all] to $100 \%$ [absolute certainty of encounter]) and were given $4 \mathrm{~s}$ for their response in order to prevent a highly controlled performance in the rating task (e.g., calculation of risk based on a thorough statistical combination of the two relative frequencies given by the forest officials). Due to this restricted 
time window it was impossible for our participants to correctly translate the mean of the relative frequencies presented in the background information into a percentage scale. Responses thus relied on the participants' gut feelings. They were given by pressing two buttons of a button box, which moved a slider across a horizontal scale. Participants were then asked to indicate the fear they experienced at the imagination of the scenario on a 17-point scale (scale $0 \%$ [no fear at all]; $100 \%$ [extreme, paralyzing fear]; manipulation check). Thus, ratings were made on a trial-bytrial basis. The 90 experimental trials were presented in random order in four runs - two blocks of 23 and two blocks of 22 trials each - that were separated by short pauses.

\section{Dependent Variables}

The dependent variables consisted of the participants' risk of encounter estimates (encounter expectancy) and their fear ratings for the three types of animals. Fear ratings served as

a manipulation check, ensuring that spider phobic individuals experienced spiders included in our studies as more threatening than snakes or birds.

\section{Experimental Design and Associated Data Analysis}

Manipulation Check (Fear Ratings). A $2 \times 3$ mixed-factorial design resulted from the manipulation of the between-subjects factor Group (spider phobic, control) and the withinsubjects factor Animal (spider, snake, bird). Significant effects as revealed in the performed analysis of variance (ANOVA) were further investigated by the use of post-hoc Tukey tests.

Encounter Expectancies (Risk Ratings). The experimental design was a $2 \times 4 \times 3$ mixedfactorial design and comprised the between factor Group (spider fearful, control) and the two within-subjects factors Objective Probability of Encounter (0-25\% [M=11\%], 26-50\% [M= 42\%], 51-75\% [M=64\%], 76-100\% [M=91\%]) and Animal (spider, snake, bird). The objective probability of an encounter in a specific trial was determined by averaging the relative frequencies of such an encounter across the two forest officials (given in the background 
information in each trial; see procedure for details). An ANOVA with the factors Group, Objective Probability, and Animal (spider, snake, bird) was conducted. On the basis of our experimental hypotheses, an a priori-specified contrast with an $\alpha$ level of .05 (one-tailed) comparing spider phobics' encounter expectancy for spiders with their encounter expectancy for snakes and birds was calculated for each probability range. Another contrast with an $\alpha$ level of .05 (two-tailed) compared the spider phobics' encounter expectancy for snakes and birds. All reported effect sizes are partial $\eta^{2}$ and are simply noted as $\eta^{2}$.

Results

\section{Fear Ratings (Manipulation Check)}

The 2 (Group) $\times 3$ (Animal) ANOVA conducted on the fear ratings showed a main effect for Group, $F(1,33)=19.59, p<.001, \eta^{2}=.37(M s=0.41$ and 0.22 , for spider fearful and control, respectively), a main effect for Animal, $F(2,66)=151.17, p<.001, \eta^{2}=.82(M \mathrm{~s}=0.58$, 0.32, and 0.03, for spiders, snakes, and birds, respectively), and a significant interaction of Group and Animal, $F(2,66)=46.41, p<.001, \eta^{2}=.58$ (Figure1a). Post-hoc Tukey tests were virtually all significant $(p s<.001)$. There was no difference in fear of birds and fear of snakes between the two groups ( $p s=1.00$ and .99 , for birds and snakes, respectively), no difference in fear of spiders versus fear of snakes in the control group $(p=1.00)$, and no difference in fear of spiders in the control group versus fear of snakes in the spider fearful group $(p=.99)$. Thus, both spider fearful and control participants displayed fear in response to phylogenetic threat (spiders and snakes) but not to birds. In addition, spider fearful individuals experienced more fear of spiders than snakes and birds, and also more fear of spiders than the control group.

Insert Figure1 about here 


\section{Encounter Expectancies}

The 2 (Group) $\times 4$ (Objective Probability) $\times 3$ (Animal) ANOVA conducted on participants' encounter expectancies yielded no significant main effect for Group, $F(1,33)=$

$0.09, n s, \eta^{2}=.00$, demonstrating that the two groups of participants did not differ in their overall average risk of encounter ratings. The remaining effects all reached significance; main effect for Objective Probability: $F(3,99)=137.90, p<.001, \eta^{2}=.81$; main effect for Animal: $F(2,66)=$ 9.34, $p<.001, \eta^{2}=.22$; interaction Group $\times$ Objective Probability: $F(3,99)=3.85, p<.05, \eta^{2}=$ .10 ; interaction Group $\times$ Animal: $F(2,66)=6.89, p<.005, \eta^{2}=.17$; interaction Objective Probability $\times$ Animal: $F(6,198)=7.58, p<.001, \eta^{2}=.19$; interaction Group $\times$ Objective Probability $\times$ Animal: $F(6,198)=2.95, p<.01, \eta^{2}=.08$. In order to better interpret this pattern of results, separate two-way ANOVAs with the factors Objective Probability and Animal were conducted for each group.

In spider fearful individuals, both main effects and the interaction effect reached significance: main effect for Objective Probability: $F(3,51)=43.55, p<.001, \eta^{2}=.72(M \mathrm{~s}=$ $0.26,0.43,0.52$, and 0.66 , for $0-25 \%, 26-50 \%, 51-75 \%$, and $76-100 \%$, respectively); main effect for Animal: $F(2,34)=9.76, p<.0005, \eta^{2}=.36(M \mathrm{~s}=0.55,0.41$, and 0.44 , for spiders, snakes, and birds, respectively); interaction of Objective Probability and Animal: $F(6,102)=8.03, p<$ $.001, \eta^{2}=.32$ (Figure1b).

To investigate the character of the interaction in spider fearful participants, separate ANOVAs were performed for each objective likelihood range. All of them yielded a significant effect for Animal (Table1). The pattern of planned comparisons showed that the spider fearful group displayed the strongest expectancy bias in the $0-50 \%$ objective probability ranges. 
Interestingly, a slight reversal of the effect was observed for the high probabilities, with birds having received higher estimates than snakes as well.

Insert Table1 about here

In the control group, only objective probabilities presented in the background information influenced the participants' risk ratings: main effect for Objective Probability: $F(3,48)=106.94$, $p<.001, \eta^{2}=.90(M \mathrm{~s}=0.18,0.39,0.52$, and 0.73 , for $0-25 \%, 26-50 \%, 51-75 \%$, and $76-100 \%$, respectively); main effect for Animal: $F(2,32)=0.36, n s, \eta^{2}=.02(M \mathrm{~s}=0.46,0.45$, and 0.45 , for spiders, snakes, and birds, respectively); interaction of Objective Probability and Animal: $F(6$, $96)=1.83, n s, \eta^{2}=.10$ (Figure1c). Figure 1 also clearly displays that both groups overestimated the low likelihoods (0-25\%) and underestimated the high likelihoods (51-100\%).

\section{Discussion}

We argue that, analogous to expectancy-value models, the perceived threat in a given situation varies as a function of both the subjective likelihood to encounter an animal and the subjective likelihood that this animal will be dangerous for the personal well-being. Such a distinction between an encounter expectancy bias and a consequences expectancy bias is critical, since these should influence different components of the fear response and, in therapy settings, it may be beneficial to address the two subcomponents separately. One strategy could focus on the reduction of fear intensity by working on the consequences of to-be-expected encounters, a second strategy on the reduction of the subjective risk of encountering the feared animals. The first strategy would thus aim at a reduction of the fear response once spiders are encountered or anticipated. The second strategy, on the contrary would aim at reducing the frequency of fear 
experiences in order to get the patient at rest and more realistically interpret low risk and safety signals in the environment.

Results of the current experiment are in line with our suggestions and underscore the importance of a closer investigation of different forms of expectancy bias in specific fear and phobia. We clearly and, for the first time directly, demonstrate that spider fearful individuals do indeed overestimate the likelihood to encounter a spider rather than a snake or a bird. Thus, our study shows that, apart from attributing greater harm to feared animals (e.g., Mühlberger et al., 2006), spider fearful individuals expect to encounter spiders more often than snakes or birds. Moreover, our experiment shows that heightened expectations for encounters are specific to spiders and that they possibly generalize across different locations (here: forest, in the de Jong and Muris, 2002, study: home environment). Importantly, the expectancy bias was present in spite of the explicit presentation of objective background information. It would be interesting to see in future research whether a similar or perhaps even more pronounced expectancy bias would emerge if a more implicit approach would be used (e.g., letting individuals refer to their own experience or using symbols/situational characteristics that increase or decrease the likelihood of encounters without being explicitly explaining these to the participant).

The difference in spider fearful individuals' subjective risk for spider encounters with respect to the other animals was impressive (on average 11 with respect to snakes and 14 with respect to birds [scale ranging from 0 to $100 \%$ ]). Thus, although spider fearful individuals in our study acknowledged that their fear of spiders was generally unfounded (according to DSM and ICD criteria), they were subject to a quite pronounced encounter expectancy bias.

A closer inspection of the encounter expectancies in the spider fearful individuals revealed a particularly strong encounter expectancy bias for spiders (when compared with snakes and spiders), when the objective background information given by the forest officials suggested 
an overall low-to-medium likelihood of an encounter. What is more, in both probability ranges this bias reflected a real overestimation of encounter expectancies for spiders with respect to the mean objective probability (see Figure 1 , dashed line for the ranges $0-25 \%$ and $26-50 \%$ ). Extreme fear of spiders might lead to the neglect of low risk background information, with fearful individuals acting according to the principle: If there is any risk at all, it is better to overestimate it and be prepared for the worst case. Interestingly, spider fearful individuals also displayed higher encounter expectancies for snakes than birds in the low objective likelihood condition. This may be explained by the fact that they more strongly feared snakes than birds. Such an interpretation is in line with work by Arntz, Rauner, and van den Hout (1995), showing that anxiety patients are characterized by a general (i.e., not specific to the domain of concern) tendency to infer danger on the basis of anxiety symptoms ("If I feel anxious, there must be danger"). ${ }^{2}$

Finally, when the objective background information given by the forest officials suggested an overall medium-to-high likelihood of an encounter, both spider fearful and controls displayed an underestimation of risk of encounter. This also included the spider fearful individuals' encounter expectancies for spiders. However, whereas no real overestimation appeared in these cases, there still was an effect of significantly higher ratings for spiders as compared with the other animals in this group of participants (cf. Table 1).

The current experiment does not allow drawing inferences whether the spider fearful participants' expectancy bias was restricted to the self or not. Although the question was to indicate the expectancy that they themselves would encounter the animals in question, it is possible that we would have obtained the same results if the fearful participants had had to give the same expectancies for the forest officials. Future experiments could investigate this issue by asking participants to specify the expectancies separately for self and others. This goes along with 
the clinical observation that phobic individuals do not generally underestimate the likelihood that specific situations could be coped with, but that they judge their personal capacities as too low to face these situations (referring to Bandura's (1977) distinction between the expectation that a specific action can lead to a desired outcome and an individual's efficacy to perform the action). 


\section{References}

Arntz, A., Rauner, M., \& van den Hout, M. (1995). “If I feel anxious, there must be danger”: Exconsequentia reasoning in inferring danger in anxiety disorders. Behaviour Research and Therapy, 33, 917-925.

Bandura, A. (1977). Self-efficacy: Toward a unifying theory of behavioral change. Psychological Review, 84, 191-215.

Beck, A. T., \& Clark, D. A. (1997). An information processing model of anxiety: Automatic and strategic processes. Behaviour Research and Therapy, 35, 49-58.

Beck, A. T., Emery, G., \& Greenberg, R. (1985). Anxiety disorders and phobias: A cognitive perspective. New York: Basic Books.

Becker, E. S., \& Rinck, M. (2004). Sensitivity and response bias in fear of spiders. Cognition and Emotion, 18, 961-976.

Dan-Glauser, E. S., \& Scherer, K. R. (in press). The Geneva Affective PicturE Database

(GAPED): A new 730 picture database focusing on valence and normative significance. Behavior Research Methods.

Davey, G. C. L. (1995). Preparedness and phobias: Specific evolved associations or a generalized expectancy bias? Behavioral and Brain Sciences, 18, 289-325.

Davey, G. C. L., \& Dixon, A. L. (1996). The expectancy bias model of selective associations:

The relationship of judgements of CS dangerousness, CS-UCS similarity and prior fear to a priori and a posteriori covariation assessments. Behaviour Research and Therapy, 34, 235252.

de Jong P., \& Merckelbach H. (1991) Covariation bias and electrodermal responding in spider phobics before and after behavioural treatment. Behaviour Research and Therapy, 29, 307314. 
de Jong, P. J., Merckelbach, H., Arntz, A., \& Nijman, H. (1992). Covariation detection in treated and untreated spider-phobic participants. Journal of Abnormal Psychology, 101, $724-727$.

de Jong, P. J., \& Muris, P. (2002). Spider phobia: Interaction of disgust and perceived likelihood of involuntary physical contact. Anxiety Disorders, 16, 51-65.

Dijk, C., \& de Jong, P. J. (2009). Fear of blushing: No overestimation of negative anticipated interpresonal effects, but a high-subjective probability of blushing. Cognitive Therapy and Research, 33, 59-74.

Gauthier, J. B., \& Bouchard, S. (1993). Adaptation canadienne-française de la version révisée du State-Trait Anxiety Inventory de Spielberger. Canadian Journal of Behavioral Sciences, 25, $559-578$.

Huijding, J., \& de Jong, P. J. (2007). Beyond fear and disgust: The role of (automatic) contamination-related associations in spider phobia. Journal of Behavior Therapy and Experimental Psychiatry, 38, 200-211.

Klorman, R., Weerts, T. C., Hastings, J. E., Melamed, B. G., \& Lang, P. J. (1974). Psychometric description of some specific-fear questionnaires, Behavior Therapy, 5, 401-409.

Mühlberger, A., Wiedemann, G., Herrmann, M. J., \& Pauli, P. (2006). Phylo- and ontogenetic fears and the expectation of danger: Differences between spider- and flight-phobic subjects in cognitive and physiological responses to disorder-specific stimuli. Journal of Abnormal Psychology, 115, 580-589.

Muris, P., Huijding, J., Mayer, B., den Breejen, E., \& Makkelie, M. (2007). Spider fear and covariation bias in children and adolescents. Behaviour Research and Therapy, 45, 26042615.

Szymanski, J., \& O'Donohue, W. T. (1995). Fear of Spiders Questionnaire. Journal of Behavior Therapy and Experimental Psychiatry, 26, 31-34. 
Tomarken, A. J., Mineka, S., \& Cook, M. (1989). Fear-relevant selective associations and covariation bias. Journal of Abnormal Psychology, 98, 381-394.

Watts, F.N. \& Dalgleish, T. (1991). Memory for phobia-related words in spider phobics. Cognition and Emotion, 5, 313-329. 
Author Note

Tatjana Aue and Marie-Eve Hoeppli, University of Geneva, Geneva, Switzerland.

This research was supported by grant PZ00P1_121590 of the Swiss National Science

Foundation to Tatjana Aue.

Correspondence concerning this article should be addressed to Tatjana Aue, Swiss Center for Affective Sciences, University of Geneva, Geneva, Switzerland. E-mail:

tatjana.aue@unige.ch. 


\section{Footnotes}

${ }^{1}$ Although our participants did meet the DSM-IV and ICD-10 criteria for spider phobia, the majority of them were not seeking for treatment. Therefore, one reviewer suggested we use the term spider fearful instead of spider phobic throughout the manuscript.

${ }^{2}$ Note, however, that a highly similar effect was also present in the control group. Although an ANOVA performed for the probability range 0-25\% in the control group just fell short of demonstrating a significant effect of Animal, $F(2,32)=2.93, p=.07, \eta^{2}=.15$, these participants displayed a tendency to indicate higher encounter expectancies for both spiders and snakes as compared with birds (cf. Figure 1c). Therefore, this result may also be explained by the concept of biological preparedness, suggesting that we are particularly prone to experience threat in response to animals having played an important role for survival in human evolution. 


\section{Figure Caption}

Figure 1: Fear and Encounter Expectancy Ratings.

Error bars depict standard errors. Scale ranging from 0 (no fear at all/no expectancy of encounter at all) to 1 (100\%; extreme, paralyzing fear/absolute certainty of encounter). Dashed lines refer to the average objective probability of encounter in each category of the factor Objective Probability. $\mathrm{N}_{\text {spider fearful }}=18 ; \mathrm{N}_{\text {control }}=17$. 


\section{Table 1}

Sub-analyses conducted on spider phobic participants' risk estimates for low, low-to-medium, medium-to-high, and high objective probabilities

Objective

Probability

\begin{tabular}{|c|c|c|c|c|c|}
\hline Range & Analysis/Contrast & $F$ & $\mathrm{df}$ & $p$ & $\eta^{2}$ \\
\hline \multicolumn{6}{|l|}{$0-25 \%$} \\
\hline & Main effect Animal & 9.96 & 2,34 & $<.001$ & .51 \\
\hline & spiders versus snakes and birds ${ }^{a}$ & 10.60 & 1,17 & $<.005$ & .55 \\
\hline & snakes versus birds ${ }^{\mathrm{b}}$ & 3.65 & 1,17 & .07 & .35 \\
\hline
\end{tabular}

$26-50 \%$

$\begin{array}{lrrrr}\text { Main effect Animal } & 15.19 & 2,34 & <.001 & .40 \\ \text { spiders versus snakes and birds }^{\mathrm{a}} & 19.09 & 1,17 & <.001 & .64 \\ & & & & \\ \text { snakes versus birds }^{\mathrm{b}} & 0.87 & 1,17 & n s & .12\end{array}$

$51-75 \%$

Main effect Animal

$5.142,34<.01$ 


$\begin{array}{lllll}\text { spiders versus snakes and birds }^{\mathrm{a}} & 7.22 & 1,17 & <.01 & .28 \\ \text { snakes versus birds }^{\mathrm{b}} & 0.84 & 1,17 & \text { ns } & .17\end{array}$

$76-100 \%$

Main effect Animal

spiders versus snakes and birds ${ }^{\mathrm{a}}$

snakes versus birds ${ }^{b}$
$5.442,34<.01$

$3.88 \quad 1,17 \quad<.05 \quad .44$

$6.94 \quad 1,17<.05 \quad .00$

Note. $N=18 ;{ }^{\mathrm{a}} p$ s are based on one-tailed testing, ${ }^{\mathrm{b}} p$ s are based on two-tailed testing 

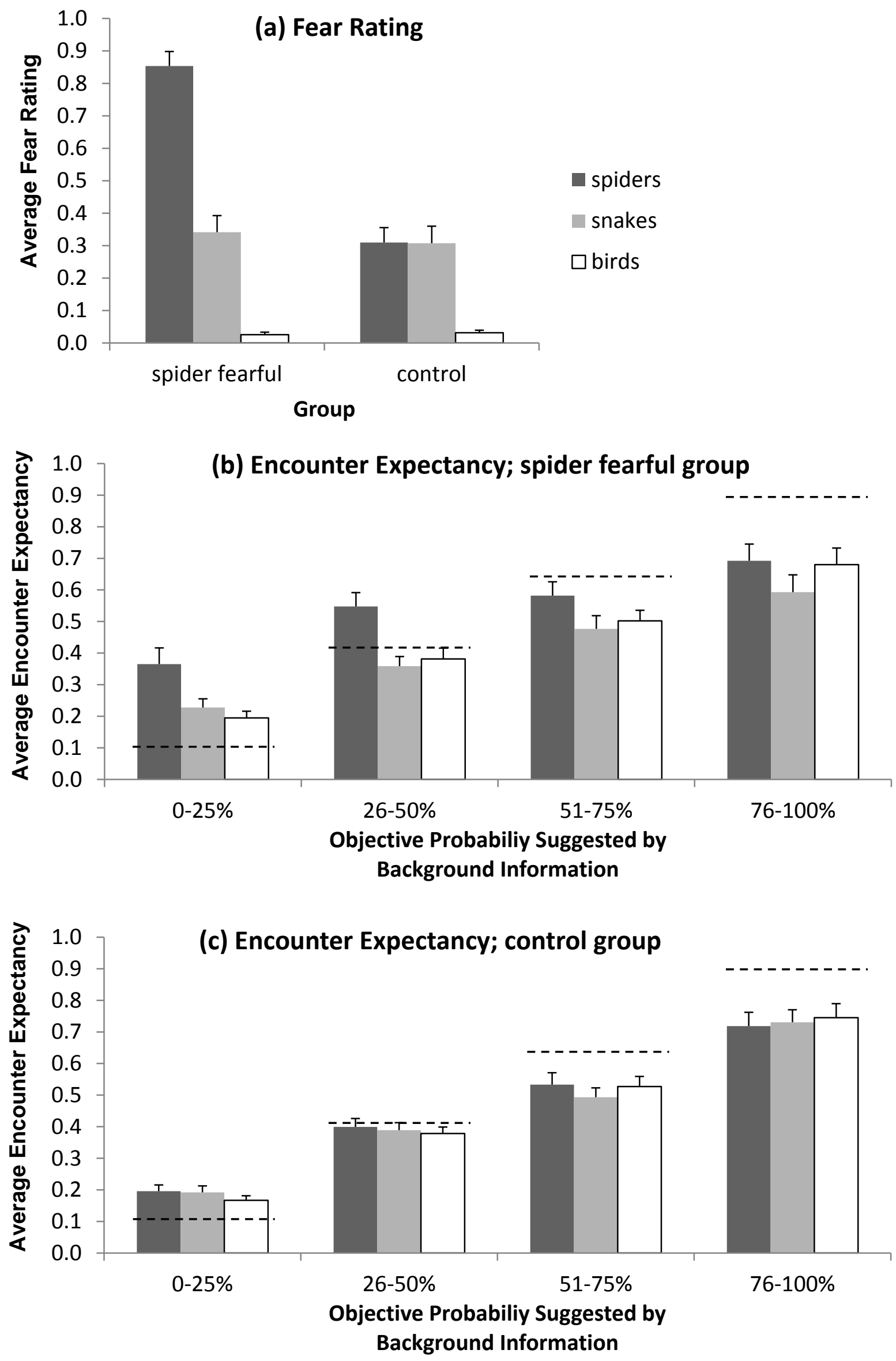